\title{
Structure and Mechanical Properties of Iron after Surface Severe Plastic Deformation under Friction with Simultaneous Nitrogen Saturation: II. The Mechanical Properties of Nano- and Submicrocrystalline Iron Saturated with Nitrogen during Deformation
}

\author{
A. I. Yurkova ${ }^{a, *}$, Yu. V. Mil'man ${ }^{b}$, and A. V. Byakova ${ }^{a, b}$ \\ ${ }^{a}$ National Technical University of Ukraine Kiev Polytechnic Institute, Kyiv, 03056 Ukraine \\ ${ }^{b}$ Institute of Problems of Materials Science, National Academy of Sciences of Ukraine, Kyiv, 03680 Ukraine \\ *e-mail:yurkova@kpm.ntu-kpi.kiev.ua
}

\begin{abstract}
The effect of structure refinement of iron to submicro- and nanograins during intense plastic deformation under friction (SPDF) with simultaneous nitrogen saturation on the mechanical characteristics (hardness, plasticity, Young's modulus) has been studied by nanoindentation. The nitrogen saturation of iron during SPDF is shown to increase the hardness of micro- and submicrocrystalline regions of the surface layer as compared to SPDF in argon as a result of solid-solution hardening. A high nitrogen concentration in an $\alpha-\mathrm{Fe}[\mathrm{N}]$ solid solution weakly affects the mechanical properties of nanocrystalline iron with grain sizes $d<$ $50 \mathrm{~nm}$, in which deformation is controlled by grain-boundary sliding.
\end{abstract}

DOI: $10.1134 / \mathrm{S} 0036029512040155$

\section{INTRODUCTION}

The influence of grain refinement to nanometer sizes on the mechanical properties of various materials, in particular, metallic alloys, is being extensively studied. Recent studies show that ultradisperse materials obtained by severe plastic deformation (SPD) exhibit unusual physical and mechanical properties as compared to polycrystalline materials [1-6]. The experimental data on the deformation behavior of materials due to refinement of grains to submicro- and nanometer-scale sizes, a decrease in Young's modulus as compared with usual polycrystalline materials, and the deviations of experimental results from the Hall and Petch concepts relative to the hardening role of grain boundaries that act like barriers preventing dislocation motion are reviewed in a number of works $[1-3,7,8]$. These deviations are provided in a certain degree by grain boundaries and triple junctions, whose volume fraction increases with decreasing grain size, and their mechanical properties differ from the properties of grains, according to, e.g., the compositional model in $[9,10]$. This model is most important for ultradisperse structures produced by SPD methods, since grain-boundary sliding and plastic rotation of grains become easier as the grain size decreases to a nanometer-scale size $[5,11,12]$.

The application of SPD to form metastable states in order to obtain high physicomechanical properties of a material by both structure refinement and a change in the phase composition due to the formation of supersaturated solid solutions is of particular interest [13-17].

The study of the structural transformations in armco iron during SPD under friction (SPDF) with simultaneous nitrogen saturation shows that the dissolution of impurity interstitial atoms during deformation favors a decrease in the size of completely stabilized nanograins [18] or the limit of deformation refinement [19], a substantial increase in the thickness of a disperse structure layer as compared to SPDF in argon, and a significant increase in the nitrogen concentration in iron as compared to the saturation with no deformation.

The aim of this work is to study the effect of refinement of iron grains to a nanometer-scale size produced by SPDF with simultaneous nitrogen saturation on its mechanical properties, such as the hardness, the plasticity, and Young's modulus.

\section{EXPERIMENTAL}

We studied samples of armco iron of $99.97 \mathrm{wt} \%$ purity with a gradient surface layer of an $\alpha-\mathrm{Fe}[\mathrm{N}]$ solid solution obtained by SPDF with simultaneous nitrogen saturation [18, 20,21]. The grain structure of the surface layer was varied from micro- to submicro- and nanometer-scale sizes. (The technique of preparation and the structure formation in iron dispersed and sat- 
urated with nitrogen are described in detail in part I of this work [22].)

In connection with a gradient structure of the surface layers and their high dispersion, the mechanical behavior of iron and a set of their mechanical characteristics (Young's modulus $E$, hardness $H_{h}$, and plasticity characteristic $\delta_{A}$ ) were found using a Nano Indenter II device equipped with a Berkovich pyramid during continuous indentation at a load of $10 \mathrm{mN}$, as in the case of iron after SPDF in a neutral medium (argon) [6]. For comparison, we also tested a sample saturated with nitrogen with no deformation during conventional furnace nitriding.

Young's modulus $E$ and hardness $H_{h}$ were calculated from the indentation depth using indenter loading curves recorded in the load $F$-indenter displacement $h$ coordinates, according to the Oliver-Pharr theory [23], which is generally accepted to find the above noted characteristics of materials [24]. In this case, we found the Meyer hardness, which is the average contact pressure on the indenter-sample contact surface and correlates well with the flow stress [25]. For each state, we processed no less than three indenter loading-unloading curves, and the results obtained were averaged.

Plasticity characteristic $\delta_{A}$ was determined according to $[24,26,27]$ and calculated from the ratio of the areas in a continuous indentation diagram as the fraction of work $A_{p}$ of plastic deformation in total indentation work $A_{t}$ by the relationship

$$
\delta_{A}=\frac{A_{p}}{A_{t}}=1-\frac{A_{e}}{A_{t}},
$$

where $A_{e}$ and $A_{t}$ are the areas under the unloading and loading curves, respectively, and $A_{p}=A_{t}-A_{e}$.

Plasticity characteristic $\delta_{A}$ found by continuous impression during nanoindentation is an analog of dimensionless plasticity parameter $\delta_{H}$ (i.e., the fraction of plastic deformation in the total elastoplastic deformation under an indenter), which characterizes the formability of a material during deformation that is retained after unloading and determined during static tests [26]. Our theoretical and experimental studies $[6,29,30]$ show that $\delta_{A} \approx \delta_{H}$ at a sufficient accuracy.

\section{MECHANICAL PROPERTIES DETERMINED BY NANOINDENTATION}

Figure 1 shows the diagrams of continuous impression measured during nanoindentation of regions with the same phase composition ( $\alpha-\mathrm{Fe}[\mathrm{N}]$ solid solution) in iron samples after SPDF with simultaneous nitrogen saturation and in the undeformed coarse-grained base metal. Table gives the mechanical properties obtained by processing of these curves. Let us consider the influence of structural factors on Young's modulus $E$, nanohardness $H_{h}$, and plasticity characteristic $\delta_{A}$.

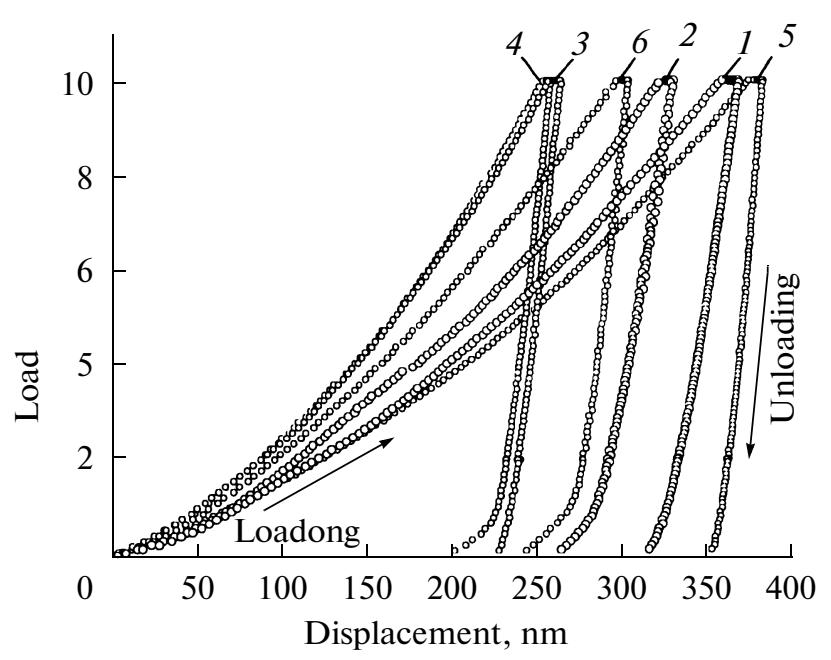

Fig. 1. Diagrams for the penetration of a pyramidal indenter during nanoindentation of armco iron produced by (1-5) SPDF with simultaneous nitrogen saturation and (6) nitrogen saturation with no deformation: (1) nanocrystalline layer, $d=10 \mathrm{~nm}\left(E=130 \mathrm{GPa}, H_{h}=3.5 \mathrm{GPa}\right)$; (2) nanocrystalline layer, $d=20 \mathrm{~nm}\left(E=168 \mathrm{GPa}, H_{h}=\right.$ $3.9 \mathrm{GPa})$; (3) nanocrystalline layer, $d=50 \mathrm{~nm}(E=$ $\left.208 \mathrm{GPa}, H_{h}=5.9 \mathrm{GPa}\right)$; (4) submicrocrystalline layer, $d=$ $200 \mathrm{~nm}\left(E=209 \mathrm{GPa}, H_{h}=6.1 \mathrm{GPa}\right) ;(5)$ coarse-grained undeformed base metal layer, $d=10 \mu \mathrm{m}(E=210 \mathrm{GPa}$, $\left.H_{h}=2.9 \mathrm{GPa}\right)$; and $(6)$ surface layer, furnace nitriding $\left(E=212 \mathrm{GPa}, H_{h}=4.4 \mathrm{GPa}\right)$.

\section{Young's Modulus}

Young's modulus $E$ of iron enriched in nitrogen with a grain size $d>30-50 \mathrm{~nm}$ almost coincides with $E$ of coarse-grained iron [31] and does not differ from the values of $E$ determined after SPDF in a neutral medium (Fig. 2a). However, as the grain size decreases further, $E$ substantially decreases, by approximately $10 \%$ at $d=20 \mathrm{~nm}$ and $30 \%$ at $d=10 \mathrm{~nm}$. In this case, the value of $E$ at $d=20 \mathrm{~nm}$ in the material after SPDF in a neutral medium and in ammonia coincide, as well as at higher values of $d$. We succeed in obtaining a finer grain $(d=10 \mathrm{~nm})$ only during SPDF with simultaneous nitrogen saturation. At this size, Young's modulus decreases most sharply. After natural aging (eight years at $293 \mathrm{~K}$ ), the supersaturated solid solution of nitrogen in iron decomposes with the precipitation of disperse $\delta^{\prime}$-phase particles (this fact was confirmed by transmission electron microscopy and microdiffraction studies); in this case, Young's modulus increases substantially (Fig. 2a, curve 2). Furnace nitriding does not change Young's modulus of $\alpha-\mathrm{Fe}$ (Fig. 2b, curve 4).

It should be noted that Fig. $2 \mathrm{~b}$ and the table show the values of Young's modulus also for the $\varepsilon\left(\mathrm{Fe}_{[2-3]} \mathrm{N}\right.$ and $\gamma^{\prime}\left(\mathrm{Fe}_{4} \mathrm{~N}\right)$ phases, which are thought are measured for the first time. 
Mechanical characteristics of various regions in the iron surface layer after SPDF with simultaneous nitrogen saturation and after furnace nitriding

\begin{tabular}{|c|c|c|c|}
\hline Phase & $E, \mathrm{GPa}$ & $H_{h}, \mathrm{GPa}$ & $\delta_{A}$ \\
\hline$\alpha-\mathrm{Fe}$ (initial state), $d=80 \mu \mathrm{m}$ & $210 \pm 10$ & $3.0 \pm 0.1$ & 0.92 \\
\hline \multicolumn{4}{|c|}{ SPDF in ammonia } \\
\hline$\alpha-\mathrm{Fe}[\mathrm{N}], d=200 \mu \mathrm{m}$ & $208 \pm 12$ & $6 \pm 0.2$ & 0.82 \\
\hline$\alpha-\mathrm{Fe}[\mathrm{N}], d=50 \mu \mathrm{m}$ & $204 \pm 10$ & $5.9 \pm 0.3$ & 0.83 \\
\hline$\alpha-\mathrm{Fe}[\mathrm{N}], d=20 \mu \mathrm{m}$ & $173 \pm 8$ & $3.7 \pm 0.4$ & 0.87 \\
\hline$\alpha-\mathrm{Fe}[\mathrm{N}], d=10 \mu \mathrm{m}$ & $128.8 \pm 10$ & $3.6 \pm 0.5$ & 0.86 \\
\hline $\mathrm{Fe}_{4} \mathrm{~N}\left(\gamma^{\prime}\right.$-phase $)$ & $172 \pm 12$ & $8.3 \pm 0.7$ & 0.65 \\
\hline \multicolumn{4}{|c|}{ SPDF in ammonia followed by natural aging } \\
\hline$\alpha-\mathrm{Fe}[\mathrm{N}], d=200 \mu \mathrm{m}$ & $207 \pm 6$ & $5.4 \pm 0.2$ & 0.84 \\
\hline$\alpha-\mathrm{Fe}[\mathrm{N}], d=50 \mu \mathrm{m}$ & $202 \pm 7$ & $5.8 \pm 0.3$ & 0.82 \\
\hline$\alpha-\mathrm{Fe}[\mathrm{N}], d=20 \mu \mathrm{m}$ & $193 \pm 12$ & $6 \pm 0.4$ & 0.78 \\
\hline$\alpha-\mathrm{Fe}[\mathrm{N}], d=10 \mu \mathrm{m}$ & $168 \pm 9$ & $6.3 \pm 0.5$ & 0.74 \\
\hline $\mathrm{Fe}_{4} \mathrm{~N}\left(\gamma^{\prime}\right.$-phase $)$ & $175 \pm 15$ & $8.7 \pm 0.9$ & 0.64 \\
\hline \multicolumn{4}{|c|}{ Furnace nitriding } \\
\hline $\mathrm{a}-\mathrm{Fe}[\mathrm{N}]$, coarse-grained & $213 \pm 14$ & $4.2 \pm 0.6$ & 0.83 \\
\hline $\mathrm{Fe}_{4} \mathrm{~N}\left(\gamma^{\prime}\right.$-phase $)$ & $170 \pm 8$ & $9.3 \pm 0.6$ & 0.62 \\
\hline $\mathrm{Fe}_{4} \mathrm{~N}(\varepsilon$-phase) & $123 \pm 15$ & $6.7 \pm 1.2$ & 0.67 \\
\hline
\end{tabular}

\section{Hardness}

The results of testing iron after SPDF with simultaneous nitrogen saturation show that hardness $H_{h}$ increases from 2.9 to $6 \mathrm{GPa}$ as the $\alpha-\mathrm{Fe}[\mathrm{N}]$ solid solution grain size decreases from coarse-grained to microand submicrocrystalline sizes $(d \approx 200 \mathrm{~nm})$ (Fig. 3). This effect is adequately described by the Hall-Petch relationship for hardness, $H=H_{0}+k_{y} d^{-0.5}$, where $k_{y}=$ $1.32 \mathrm{MGf} \mathrm{m}^{1 / 2}$, which falls in the range $\left(k_{y}=1.26-\right.$ 2.28) presented in [32,33] for usual polycrystalline $\alpha-\mathrm{Fe}\left(k_{y}\right.$ determined by hardness measurements is assumed to be higher by a factor of three than that determined by yield strength measurements). As the grain decreases further (from 200 to $50 \mathrm{~nm}$ ), the hard-


Fig. 2. (a) Young's modulus of armco iron vs. grain size $d^{-0.5}$ and (b) Young's modulus across the surface layer thickness after various treatments: (1) SPDF with simultaneous nitrogen saturation, (2) SPDF with simultaneous nitrogen saturation and subsequent natural aging, (3) SPDF in argon, and (4) furnace nitriding. 

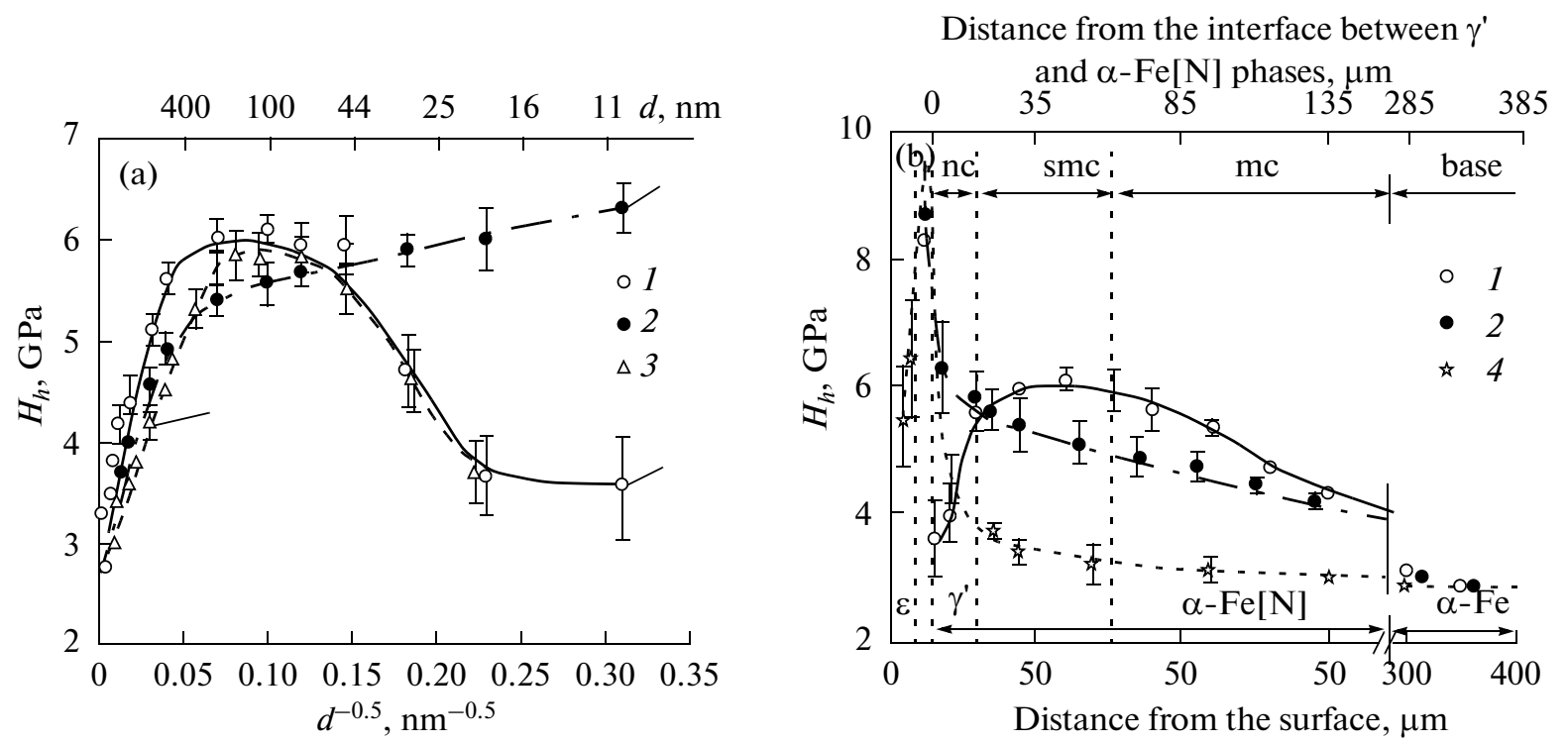

Fig. 3. (a) Hardness of armco iron vs. grain size $d^{-0.5}$ and (b) hardness across the surface layer thickness after various treatments: (1) SPDF with simultaneous nitrogen saturation, (2) SPDF with simultaneous nitrogen saturation and subsequent natural aging, (3) SPDF in argon, and (4) furnace nitriding.

ness is unchanged and remains high, which is a deviation from the Hall-Petch relationship, and the hardness substantially decreases in the layer region with grain sizes $d<50 \mathrm{~nm}$. In this case, the hardness of the layer region of the $\alpha-\mathrm{Fe}[\mathrm{N}]$ solid solution with grain sizes of $20-50 \mathrm{~nm}$ obtained after SPDF with simultaneous nitrogen saturation almost coincides with the hardness after SPDF in argon [6] for the same grain size. As the grain size decreases to $10 \mathrm{~nm}$, the hardness of nanocrystalline nitrogen ferrite is almost unchanged $(3.6 \pm 0.5 \mathrm{GPa})$.

After natural aging of the samples treated by SPDF with simultaneous nitrogen saturation, the hardness of the nanocrystalline region of the $\alpha-\mathrm{Fe}[\mathrm{N}]$ phase substantially increases and reaches $6.3 \pm 0.5 \mathrm{GPa}$ for a grain size of $10 \mathrm{~nm}$ (Fig. 3a, curve 2).

The hardness is maximum, $8.7 \mathrm{GPa}$, on the sample surface, i.e., in the $\mathrm{Fe}_{4} \mathrm{~N}$ ( $\gamma^{\prime}$ phase) layer (Fig. 3b, table). It should be noted that, despite the method by which the layer of $\mathrm{Fe}_{4} \mathrm{~N}$ cubic nitride was formed (SPDF in ammonia, nitrogen saturation with no deformation, or as a result of subsequent aging), its hardness is the same (within the scatter of the experimental data) and significantly exceeds the hardness of hexagonal nitride $\mathrm{Fe}_{2}-3 \mathrm{~N}$ ( $\varepsilon$ phase).

\section{Plasticity Characteristic}

The dependence of plasticity characteristic $\delta_{A}$ on the grain size and the distribution of its values over the surface layer thickness reflect the character of the hardness distribution: $\delta_{A}$ decreases with increasing hardness (Figs. 3, 4). In the $\alpha-\mathrm{Fe}[\mathrm{N}]$ solid solution, parameter $\delta_{A}$ decreases with decreasing grain size to
$200 \mathrm{~nm}$ and, then, levels off; the plasticity parameter increases as the grain size smaller $50 \mathrm{~nm}$. In addition, parameter $\delta_{A}$ of the $\alpha-\mathrm{Fe}[\mathrm{N}]$ solid solution with grain sizes $d<50 \mathrm{~nm}$ is higher $(0.86-0.87)$ as compared to $\delta_{A}=0.83$ of the coarse-grained $\alpha-\mathrm{Fe}[\mathrm{N}]$ phase obtained after nitrogen saturation upon furnace nitriding, and it substantially exceeds the plasticity characteristics of the nitride $\mathrm{Fe}_{4} \mathrm{~N}$ layer (table).

It should be noted that the values of $\delta_{A}$ of the nanocrystalline region in the samples aged after SPDF with simultaneous nitrogen saturation are significantly lower than those the as-prepared samples (Fig. 4). The effect of decreasing $\delta_{A}$ with increasing hardness due to second-phase particles enhances the simultaneous decrease in Young's modulus (see [26] and Fig. 2).

The dispersion of the grain structure during SPDF from the micro- to submicro- and nanosized level changes the hardness and decreases $\delta_{A}$ below a critical value of 0.9 . According to [26], bulk samples undergo brittle fracture during tensile tests at $\delta_{A}<0.9$. However, the probability of brittle fracture of thin surface layers is substantially lower, since such layers are deformed in the elastic region during bending [34].

\section{DISCUSSION OF THE RESULTS}

Using the nanoindentation method, it was stated in [6] that the hardness of fcc metals only increases and plasticity decreases with decreasing the grain sizes to $20 \mathrm{~nm}$. The opposite effect, namely, a decrease in the hardness (from 5.8 to $3.7 \mathrm{GPa}$ ) and an increase in the plasticity parameter $\delta_{A}$ (from 0.82 to 0.87 ) has been revealed in bcc iron produced by SPDF in a neutral atmosphere (argon) as the grain size decreases from 50 

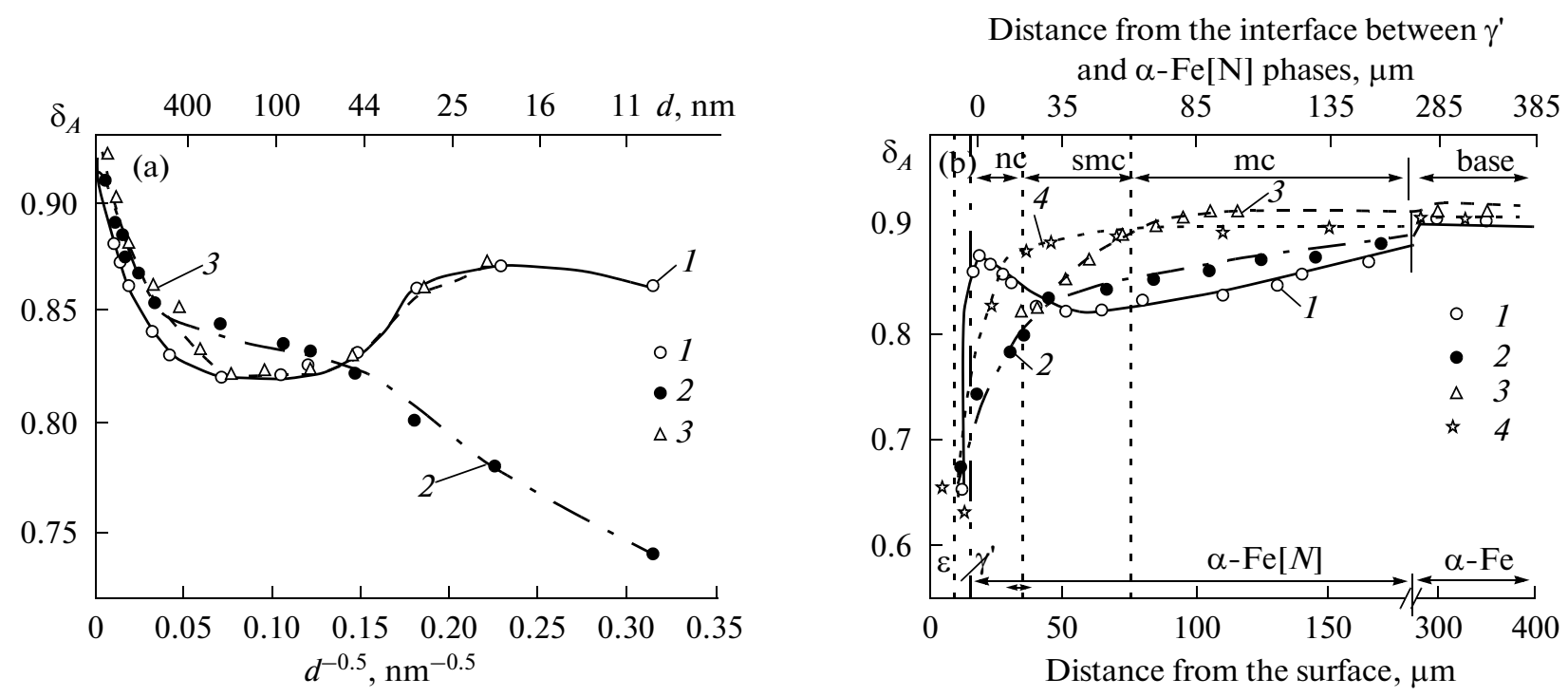

Fig. 4. (a) plasticity characteristic $\delta_{A}$ of armco iron vs. grain size $d^{-0.5}$ and (b) $\delta_{A}$ across the surface layer thickness after various treatments: (1) SPDF with simultaneous nitrogen saturation, (2) SPDF with simultaneous nitrogen saturation and subsequent natural aging, (3) SPDF in argon, and (4) furnace nitriding.

to $20 \mathrm{~nm}$. It was also shown that Young's modulus decreases beginning from grain sizes $d<30 \mathrm{~nm}$, and it decreases by $10 \%$ at a grain size of $20 \mathrm{~nm}$ as compared to a usual polycrystalline state.

The decrease in Young's modulus $E$ with grain refinement is almost the same for SPDF in both ammonia and a neutral atmosphere. It allows the assumption that similar behavior of $E$ is provided by structural factors and not related to the presence of nitrogen. In nanocrystalline iron, a significant part of atoms is located in the intergranular space (in grain boundaries (GBs) and triple junctions (TJs)). To estimate the amount of such atoms, we consider a simple model of a crystal consisting of nanometer-sized grains of a cubic shape (Fig. 5). We denote the grain size by $d$ and the boundary width by $h$. The number of

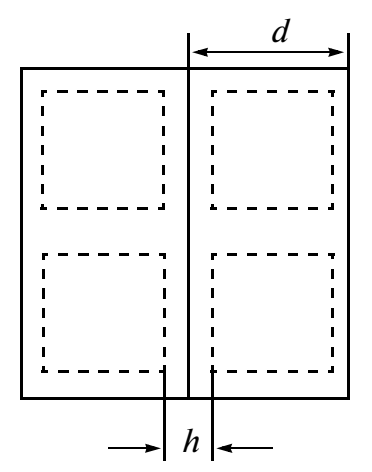

Fig. 5. Model of an iron crystal for estimating the volume fraction of atoms located in the intergranular space (GBs, TJs) after SPDF. atoms in a grain of a bcc crystal can be found from the expression $N=\left(\frac{2 d}{a}\right)^{3}$, where $a$ is the lattice parameter. The number of atoms in one grain that do not belong to the intergranular space (GBs and TJs) is $N_{1}=\left(\frac{2 d}{a}-h\right)^{3}, h$ is measured in terms of the number of atoms belonging to the boundary. Then, the volume fraction of atoms in the intergranular space (in GBs and TJs) is determined as $f=\frac{N-N_{1}}{N}$.

It follows from the dependence of Young's modulus on the volume fraction of atoms in GBs and TJs (intergranular space) at various $h$ that a larger part of atoms in a nanocrystalline material is in a slightly ordered state in GBs and TJs (Fig. 6). This fact and the microporosity that form during grain-boundary sliding upon plastic deformation of nanocrustalline iron are likely to be responsible for such a strong (to 30\%) decrease in Young's modulus in a nanocrystalline structure with $d=10 \mathrm{~nm}$.

In a number of works, it is shown that Young's modulus of nanomaterials obtained by SPD methods decreases because of the formation of a crystallographic deformation texture [2]. However, X-ray diffraction and electron microscopy reveals chaotic crystallographic orientations of grains, and there is no crystallographic texture in iron after SPDF. The increase in Young's modulus during natural aging of the nanocrystalline iron (Fig. 2) is likely to be related to the improvement of the crystal structure near GBs 

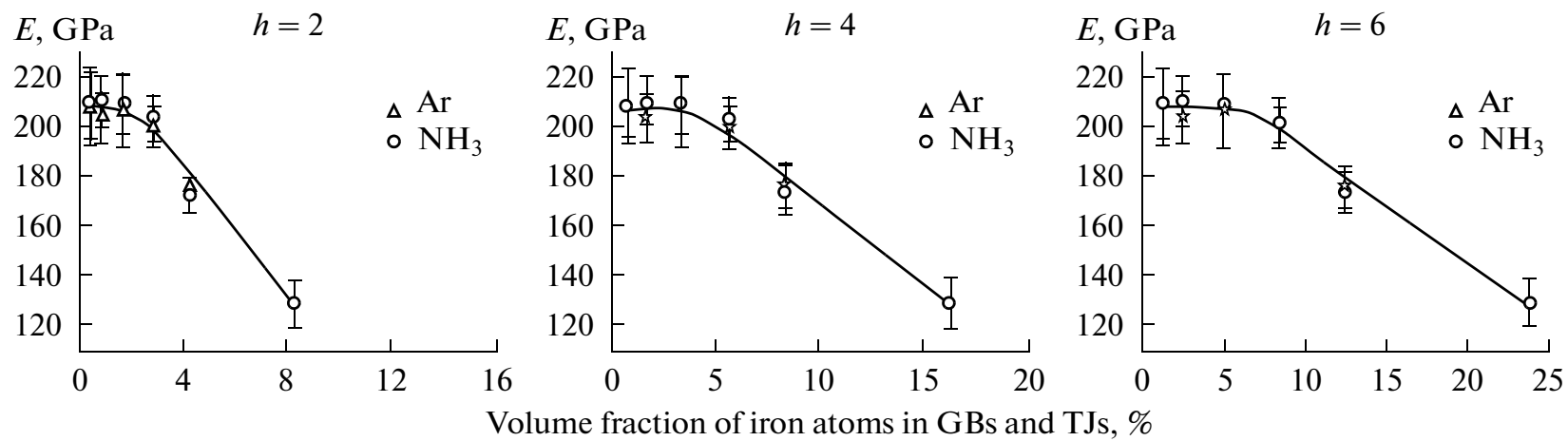

Fig. 6. Dependence of Young's modulus on the fraction of iron atoms in GBs and TJs (in the intergranular space) after SPDF in ammonia $\left(\mathrm{NH}_{3}\right)$ and after SPDF in argon (Ar) for various values of boundary width $h$ ( $h$ is measured in terms of the number of atoms belonging to the boundary).

and TJs and indicates a metastbility of the nanocrystalline state at room temperature.

As follows from the results shown in Fig. $3 \mathrm{a}$, the deviation from the Hall-Petch relationship in bcc $\alpha$-Fe saturated with nitrogen during SPDF (curve 1), as that in $\alpha-F e$ after SPDF in argon (curve 3), is observed at larger grain sizes $(d \leq 200 \mathrm{~nm})$ as compared to fcc metals, the hardness of which slightly decreases

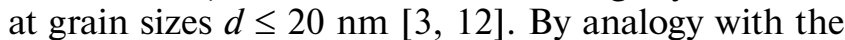
results for $\alpha$-Fe dispersed during SPDF in a neutral atmosphere (argon) [6], these data indicate a deviation from the Hall-Petch relationship and a change in the deformation mechanism when the addition of grain-boundary sliding to the relay-race mechanism of dislocation motion through GBs becomes energetically favorable. In the [alpha]-Fe[N] layer region with grain sizes from 200 to $50 \mathrm{~nm}$, deformation occurs by a mixed mechanism: grain-boundary sliding is added to a usual dislocation mechanism. At a grain size $d<$ $50 \mathrm{~nm}$ (when the hardness decreases with decreasing grain size), the dominant role belongs to grain-boundary sliding; in this case, rotation deformation modes are activated $[35,36]$, and deformation mainly occurs via grain-boundary sliding and nanograin rotation, which is caused by high stresses in nanograin TJs.

After natural aging of the samples treated by SPDF with simultaneous nitrogen saturation (Fig. 3), the hardness of the nanocrystalline $\alpha-\mathrm{Fe}[\mathrm{N}]$ phase with grain sizes $d<50 \mathrm{~nm}$ increases substantially because of the existence of second-phase particles. The nanosized $\mathrm{Fe}_{4} \mathrm{~N}$ ( $\gamma^{\prime}$ phase) particles that precipitate during natural aging from a supersaturated $\alpha-\mathrm{Fe}[\mathrm{N}]$ solid solution strengthen GBs and TJs with a decreased strength. In this case, the effect of the geometric factor is changed to the opposite effect, increasing the hardness of nitrogen ferrite despite its nanocrystalline state. It should be noted that the hardening of the nanocrystalline region of the $\alpha-\mathrm{Fe}[\mathrm{N}]$ solid solution with grain sizes $d<50 \mathrm{~nm}$ after natural aging is accompanied by a decrease in the thickness of the nanocrystalline region with grain sizes $d>50 \mathrm{~nm}$, as well as sub- micro- and microcrystalline regions. This effect can be explained by the redistribution of nitrogen atoms between the nanocrystalline region adjacent to the surface and more distant regions of the gradient layer (i.e., by the diffusion of nitrogen atoms to the surface to compensate the precipitation of nitrogen from an $\alpha-\mathrm{Fe}[\mathrm{N}]$ solid solution to form $\gamma^{\prime}$ phase nanoparticles).

We call attention to the fact that, despite a higher nitrogen content in the samples after SPDF with simultaneous nitrogen saturation as compared to the samples after nitrogen saturation with no deformation (furnace nitriding), the hardness of the nanocrystalline $\alpha-\mathrm{Fe}[\mathrm{N}]$ solid solution with grain sizes $d<50 \mathrm{~nm}$ is lower than that of iron after furnace nitriding near the interface with the $\gamma^{\prime}$ phase (Fig. 3b, table). In this case, the hardness of nanocrystalline $\alpha-\mathrm{Fe}[\mathrm{N}]$ with grain sizes $d<50 \mathrm{~nm}$ is almost half that after aging (Fig. 3). Since the hardness of nitrogen ferrite $(\alpha-\mathrm{Fe}[\mathrm{N}]$ solid solution) is determined by two parameters (the grain size and the nitrogen content in it), it is evident that solid-solution strengthening is suppressed by the size effect after SPDF with simultaneous nitrogen saturation.

The results of this study show that the nitrogen saturation of iron during SPDF increases the hardness of the micro- and submicrostructure regions in the surface layer as compared to SPDF in argon due to solidsolution strengthening. In these regions, a dislocation mechanism operates.

The decrease in the hardness of the $\alpha-\mathrm{Fe}[\mathrm{N}]$ phase with decreasing grain sizes below $50 \mathrm{~nm}$ increases plasticity characteristic $\delta_{A}$ (to 0.87 ) of the nanocrystalline nitrogen-containing iron produced by SPDF with simultaneous nitrogen saturation despite a somewhat decrease in Young's modulus (Fig. 2). It is due to the fact that the hardness decreases more quickly than Young's modulus and that $\delta_{A}$ is mainly determined by the $H_{M} / E$ ratio $[26,29]$. Essentially, the dispersion of bcc iron to the nanocrystalline state $(d<50 \mathrm{~nm})$ is accompanied by an increase in the plasticity charac- 
teristic, while the dispersion of the grain structure of fcc metals to $20 \mathrm{~nm}$ only decreases the plasticity [6].

A high nitrogen concentration in the $\alpha-\mathrm{Fe}[\mathrm{N}]$ solid solution weakly influences the mechanical properties of nanocrystalline iron, since deformation is controlled by grain-boundary sliding. The influence of nitrogen (with no second-phase precipitates) manifests itself in the thickening of a nanocrystalline layer with high plasticity characteristics (Fig. 4b). The simultaneous decrease in the hardness and Young's modulus allows one to retain high plasticity characteristics in the nanocrystalline iron produced by SPDF as the grain size decreases below $50 \mathrm{~nm}$ (Fig. 4). The nanocrystalline iron produced by SPDF in ammonia or in a neutral atmosphere (argon) [6] demonstrates high plasticity characteristic $\delta_{A}$ as compared to parameter $\delta_{A}$ after nitrogen saturation without deformation and after SPDF in ammonia followed by aging, where nitride-phase precipitates exist (Fig. 4).

\section{CONCLUSIONS}

(1) As the $\alpha-\mathrm{Fe}[\mathrm{N}]$ solid solution grains obtained by SPDF with simultaneous nitrogen saturation decrease to sizes smaller than $30 \mathrm{~nm}$, Young's modulus decreases as compared to the coarse-grained state. The effect of a decrease in the elasticity modulus increases to $30 \%$ when the grain size decreases to $10 \mathrm{~nm}$, whereas $E$ of $\alpha-\mathrm{Fe}[\mathrm{N}]$ with grain sizes of $20 \mathrm{~nm}$ decreases by $10 \%$ and corresponds to Young's modulus for $\alpha$-Fe with the same grain size after refinement by SPDF in argon. The decrease in $E$ in the material in the nanocrystalline state is due to specific features of its structure rather than to a high nitrogen content in an $\alpha-\mathrm{Fe}[\mathrm{N}]$ solid solution.

(2) As compared to SPDF in argon, the nitrogen saturation during SPDF increases the hardness in micro- and submicrocrystalline regions of an $\alpha-\mathrm{Fe}[\mathrm{N}]$ solid solution, in which the dislocation mechanism of deformation operates.

(3) A high nitrogen concentration in an $\alpha$ solid solution weakly influences the mechanical properties (hardness, plasticity characteristic $\delta_{A}$, Young's modulus) of nanocrystalline $(d<50 \mathrm{~nm})$ nitrogen-containing iron, since deformation in the nanocrystalline state is controlled by grain-boundary sliding.

(4) The influence of nitrogen (in the absence of second-phase precipitates) manifests itself in an increase in the thickness of the disperse-structure layer with high plasticity characteristic $\delta_{A}$. In this case, Young's modulus, the hardness, and the plasticity are retained at the level characteristic of $\alpha$-Fe after SPDF in a neutral atmosphere (at the same grain sizes).

(5) In nanocrystalline $\alpha-\mathrm{Fe}[\mathrm{N}]$ produced by SPDF with simultaneous nitrogen saturation, as well as in $\alpha-\mathrm{Fe}$ after SPDF in argon, the hardness decreases (from 6.0 to $3.6 \mathrm{GPa}$ ) and the plasticity parameter $\delta_{A}$ increases (from 0.82 to 0.87 ) when the grain size decreases from 50 to $20 \mathrm{~nm}$, while a decrease in the grain sizes of fcc metals to $20 \mathrm{~nm}$ increases the hardness and decreases the plasticity.

(6) The precipitation of disperse particles of the $\gamma^{\prime}$ phase (nitride $\mathrm{Fe}_{4} \mathrm{~N}$ ) during natural aging of nanocrystalline $(d<50 \mathrm{~nm})$ iron with a high nitrogen concentration radically changes its mechanical properties. In a layer region with a grain size of $20 \mathrm{~nm}$, the hardness increases to 6.3. GPa, plasticity characteristic $\delta_{A}$ decreases to 0.78 , and Young's modulus increases to the values characteristic of the coarsegrained state.

\section{ACKNOWLEDGMENTS}

We are grateful to S.N. Dub for performing the nanoindentation tests.

This work was supported in part by the Ministry of Education and Science of Ukraine (project no. 2211-f) and the National Academy of Sciences of Ukraine (project no. III-14-07).

\section{REFERENCES}

1. R. Z. Valiev and I. V. Aleksandrov, Nanostructural Materials Produced by Severe Plastic Deformation (Logos, Moscow, 2000).

2. P. A. Andrievskii and A. M. Glezer, "Size Effects in 1 Nanocrystalline Materials: 2. Mechanical and Physical Properties," Phys. Met. Metallogr. 89 (1) 83-103 (2000).

3. N. I. Noskova and P. P. Mulyukov, Submicrocrystalline and Nanocrystalline Metals and Alloys (UrO RAN, Yekaterinburg, 2003).

4. Yu. R. Kolobov, R. Z. Valiev, G. P. Grabovetskaya, et al., Grain-Boundary Diffusion and the Properties of Nanostructured Materials (Nauka, Novosibirsk, 2001).

5. R. Z. Valiev, Y. Estrin, Z. Horita, T. G. Langdon, et al., "Producing Bulk Ultrafine-Grained Materials by Severe Plastic Deformation," Nanostructured Materials, 33-39 (April, 2006).

6. A. I. Yurkova, Yu. V. Milman, and A. V. Byakova, "Structure and the Mechanical Properties of Iron after Surface Severe Plastic Deformation under Friction: II. The Mechanical Properties of Nano- and Submicrocrystalline Iron," Deform. Razrushenie Mater., No. 2, 2-8 (2009).

7. K. S. Kumar, H. Van Swygenhoven, and S. Suresh, "Mechanical Behavior of Nanocrystalline Metals and Alloys," Acta Mater. 51, 5743-5774. (2003).

8. A. P. Shpak, Yu. A. Kunitskii, and V. L. Karbovskii, Cluster and Nanostructured Materials, Vol. 1 (Akademperiodika, Kiev, 2001).

9. H. Mughrabi, "Dislocation Wall and Cell Structure and Long-Range Internal Stresses in Deformed Metal Crystals," Acta Metall. 32, 1367-1379 (1983).

10. T. Ungar, H. Mughraby, D. Rönnpagel, and M. Wilkens, "X-Ray Line-Broadening Study of the Dislocation Cell Structure in Deformed [001]-Oriented Copper Single Crystals,” Acta Metall. 32, 333-342 (1984). 
11. A. M. Glezer, "Strength and Plasticity of Nanocrystals," in Proceedings of the 1st International Conference on Deformation and Fracture of Materials, Moscow (Moscow, 2006), pp. 14-16.

12. H. Van Swygenhoven and J. R. Weertman, "Deformation in Nanocrystalline Metals," Materals Today 9 (5), 24-31 (2006).

13. R. Z. Valiev, R. K. Islamgaliev, and I. V. Alexandrov, "Bulk Nanostructured Materials Processed by Severe Plastic Deformation,” Progr. Mater. Sci. 45, 103-109 (2000),

14. V. V. Stolyarov and R. Z. Valiev, "Production of Ultrafine-Grained Alloys by Equal-Channel Angular Pressing," Russian Metallurgy (Metally), No. 2, 5-11 (2004).

15. V. V. Stolyarov, R. Lapovok, I. G. Drodova, and P. F. Thomson, "Ultrafine-Grained $\mathrm{Al}-15$ wt \% Fe Alloy Processed by ECAP with Backpressure," Mater. Sci. Eng. A 357, 159-167 (2003).

16. W. Lojkowski, Yu. V. Milman, S. I. Chugunova, et al., "The Mechanical Properties of the Nanocrystalline Layer on the Surface of Railway Tracks," Mater. Sci. Eng. A 303, 209-215 (2001).

17. A. Yurkova, A. Belots'ky, A. Byakova, Yu. Milman, and S. Dub, "Mechanical Behavior of Nanostructured Iron Fabricated by Severe Plastic Deformation under Diffusion Flow of Nitrogen," Mater. Sci. Forum 503-504, 645-650 (2006).

18. A. I. Yurkova, Yu. V. Milman, and A. V. Byakova, "Structure and the Mechanical Properties of Iron after Surface Severe Plastic Deformation under Friction: I. Specific Features of Formation of the Structure," Deform. Razrushenie Mater., No. 1, 2-11 (2009).

19. V. I. Kopylov and V. N. Chuvul'deev, "Grain Refinement Limit upon Equal-Channel Angular Deformation," Russian Metallurgy (Metally), No. 1, 22-35 (2004).

20. A. Yurkova, A. Belots'ky, and A. Byakova, "Anomalous Nitrogen Solubility in Gradient Nanostructured Layer Formed in the Surface of Bulk Iron by Severe Plastic Deformation under Friction," in Nanostructured Materials by High-Pressure Severe Plastic Deformation (Springer, Netherlands, 2005), Vol. 212, pp. 107-112.

21. A. Yurkova, A. Belots'ky, Yu. V. Mil'man, and A. Byakova, "Formation of Nanostructure on the Surface of Iron under Friction," Nanofizika, Nanosistemy, Nanomaterialy 2 (2), 633-644 (2004).

22. A. I. Yurkova, Yu. V. Milman, and A. V. Byakova, "Structure and the Mechanical Properties of Iron after Surface Severe Plastic Deformation under Friction: I. Specific Features of Formation of the Structure," Deform. Razrushenie Mater., No. 2, 1-8 (2011).
23. W. C. Oliver and G. M. Pharr, "An Improved Technique for Determining Hardness and Elastic Modulus Using Load and Displacement Sensing Indentation Experiments," Mater. Res. 7 (6), 1564-1583 (1992).

24. ISO14577-1: 2002. Metallic Materials. Instrumented Indentation Test for Hardness and Materials Parameters. Test Method.

25. D. Tabor, Hardness of Metals (Clarendon Press, Oxford, 2000), Preprint 1951.

26. Yu. V. Milman, V. A. Galanov, and S. I. Chugunova, "Plasticity Characteristics Obtained through Hardness Measurement," Acta Metall. Mater. 41 (9), 2523-2532 (1993).

27. Yu. V. Milman, S. I. Chugunova, and I. V. Goncharova, "Plasticity Characteristic Obtained by Indentation Technique for Crystalline and Noncrystalline Materials in Wide Temperature Range," High Temperature Materials and Processes 25 (1-2), 39-46 (2006).

28. A. Byakova, Yu. Milman, and A. Vlasov, "A High Performance Ceramic Coatings for Cutting Tool: Perspective in Improvement of Coating Mechanical Properties," in Proceedings of 8th CIRP International Workshop on Modeling of Machining Operations, Chemnitz, Germany (2005), pp. 559-568.

29. Yu. V. Milman, "Plasticity Characteristic Obtained by Indentation,” J. Appl. Phys. D 41, 1-9 (2008).

30. Yu. Milman, S. Dub, and A. Golubenko, "Plasticity Characteristic Obtained through Instrumental Indentation," Mater. Res. Symp. Proc. 1049, 121-128 (2008).

31. I. N. Frantsevich, F. F. Voronov, and S. A. Bakuta, Elastic Constants and Elastic Moduli of Metals and Nonmetals: A Handbook (Naukova Dumka, Kiev, 1982).

32. V. I. Trefilov, V. F. Mouseev, E. P. Pechkovskii, et al., Strain Hardening and Fracture of Polycrystalline Materials (Naukova Dumka, Kiev, 1987).

33. V. I. Trefilov, Yu. V. Milman, and S. A. Firstov, Physical Basics of the Strength of High-Temperature Materials (Naukova Dumka, Kiev, 1975).

34. A. Byakova, Yu. Milman, and A. Vlasov, "Application of the Plasticity Characteristic Determined by the Indentation Technique for Evaluations of Mechanical Properties of Coatings: Part II. Guidelines to Coating Development and Processing Control," Science of Sintering 36, 93-104 (2004).

35. N. I. Noskova, "Structural Features and Mechanisms of Deformation of Nanocrystalline Materials," J. Phys. Metals and Metallography, Suppl. 94, 119-130 (2002).

36. V. A. Pozdnyakov, "Mechanisms of Plastic Deformation and the Anomalies of the Hall-Petch Dependence in Nanocrystalline Metallic Materials," Phys. Met. Metallogr. 96 (1), 114-128 (2003).

SPELL: 1. ok 